\title{
Diverticulitis aguda complicada: tendencias en el tratamiento actual*
}

\author{
Drs. NICOLÁS PEREIRA C. ${ }^{1}$, JAVIER VEGA S. ${ }^{2}$, ALEJANDRO READI V. ${ }^{1}$, \\ MARIO ABEDRAPO M. ${ }^{1}$, INT. ALFONSO GALLEGUILLOS G. ${ }^{3}$ \\ 1 Departamento de Cirugía, Hospital Clínico Universidad de Chile, Santiago. \\ 2 Mutual de Seguridad de la Cámara Chilena de la Construcción, Antofagasta. \\ 3 Facultad de Medicina, Universidad de Chile, Santiago. \\ Chile.
}

\begin{abstract}
Acute complicated diverticulitis. New trends in treatment

Diverticular disease refers to the presence of colon diverticula. Its prevalence increases with age and in a few cases present as diverticulitis. Acute complicated diverticulitis is potentially mortal, since it can perforate and require emergency surgery. The treatment of choice is the excision of the perforated segment and the creation of a proximal colostomy. There are other therapeutic alternatives such as excision with primary anastomosis and novel approaches such as laparoscopic peritoneal lavage. We herein review the new treatments of acute diverticulitis.
\end{abstract}

Key words: Diverticular disease, diverticulitis, colectomy, laparoscopic lavage.

\section{Resumen}

La enfermedad diverticular se refiere a la presencia de divertículos en el colon. Su prevalencia aumenta con la edad y sólo en pocos casos se presenta como diverticulitis aguda. La diverticulitis aguda complicada es una condición potencialmente mortal, por la posibilidad de una perforación libre que requiera cirugía de urgencia. El tratamiento de elección en la mayoría de los casos es la resección del segmento colónico que incluya la perforación y la creación de una colostomía proximal. Existen otros tratamientos en el manejo quirúrgico de la diverticulitis perforada, como la resección con anastomosis primaria y nuevas aproximaciones, como el lavado peritoneal por vía laparoscópica. Se revisa y discute en este artículo la tendencia actual en el manejo de la diverticulitis aguda perforada.

Palabras clave: Enfermedad diverticular, diverticulitis aguda, colectomía, lavado peritoneal laparoscópico.

\footnotetext{
Los autores no declaran conflictos de interés.

Correspondencia: Dr. Nicolás Pereira C.

Santos Dumont 999, Santiago, Chile.

nicolaspereirac@gmail.com
}

*Recibido el 24 de junio de 2012 y aceptado para publicación el 26 de julio de 2012. 


\section{Introducción}

La enfermedad diverticular (ED) puede ser asintomática o sintomática. Esta última, puede dividirse en complicada o no complicada. Nos referimos a ED complicada cuando hay presencia de diverticulitis aguda, abscesos, obstrucción, estenosis, fistulas, hemorragia o perforación.

La prevalencia de la ED es menor a un $2 \%$ en menores de 30 años, mayor del $40 \%$ en mayores de 60 años y alrededor del $66 \%$ en mayores de 85 años $^{1,2}$. La diverticulitis aguda es la complicación más frecuente, afectando entre el 10 y el $25 \%$ de los pacientes $^{3-5}$; en el mundo occidental es responsable de un gran número de hospitalizaciones ${ }^{6}$. Entre el $15 \%$ y el $30 \%$ de los pacientes con diverticulitis aguda requiere una intervención quirúrgica ${ }^{7,8}$.

Además de clasificar la ED, es apropiado subdividir la diverticulitis aguda en complicada y no complicada, y la primera de éstas en diferentes categorías mediante la tomografía axial computarizada (TC) ${ }^{9}$, ya que la morbilidad y mortalidad de esta condición es muy variable. Tradicionalmente, la clasificación más usada ha sido la de Hinchey, que divide la diverticulitis aguda complicada en subgrupos, basado en el grado y extensión del proceso inflamatorio-infeccioso (Tabla 1).

El tratamiento depende de la gravedad del cuadro y de la condición basal del paciente. En relación a la diverticulitis aguda no complicada, los pacientes jóvenes, inmunocompetentes, sin complicaciones asociadas, pueden ser manejados en forma ambulatoria, con antibióticos orales. La antibioticoterapia debe abarcar a los microorganismos Gram negativos aerobios y anaerobios. Se les debe realizar un seguimiento para evaluar la respuesta al tratamiento, pueden llegar a requerir hospitalización si los síntomas no mejoran. Aquellos que experimenten recurrencia deben ser intervenidos quirúrgicamente.

Hoy se recomienda el tratamiento quirúrgico después del primer episodio de diverticulitis aguda complicada, debido a que un tercio de los pacientes presentará un segundo episodio ${ }^{10}$. Sin embargo, el manejo no quirúrgico puede ser apropiado en un grupo seleccionado de pacientes si la edad o las

Tabla 1. Clasificación de Hinchey ${ }^{9}$

\begin{tabular}{|cl|}
\hline Hinchey & Hallazgos \\
I & Absceso o flegmón pericólico \\
II & $\begin{array}{l}\text { Absceso pélvico, intraabdominal o retro- } \\
\text { peritoneal }\end{array}$ \\
III & Peritonitis purulenta generalizada \\
IV & Peritonitis estercorácea generalizada \\
\hline
\end{tabular}

comorbilidades médicas no permiten una cirugía segura $^{11}$.

\section{Alternativas de manejo quirúrgico para la diverticulitis aguda perforada}

La perforación libre en la diverticulitis es un escenario de riesgo vital que requiere una intervención quirúrgica inmediata. El tratamiento de elección en la mayoría de los casos es la resección del segmento colónico que incluya la perforación y la creación de una colostomía proximal. Muchos autores se refieren a ésta como cirugía de Hartmann, que por definición incluye la resección del sigmoides, cierre del muñón rectal y creación de una colostomía terminal, lo cual puede hacerse por vía laparoscópica ${ }^{12,13}$. Algunos cirujanos han sugerido que, especialmente en pacientes cursando con una sepsis severa y que se encuentran hemodinámicamente inestables, el objetivo inicial debiera ser una resección expedita limitada al segmento comprometido ${ }^{14}$, a veces llamada "perforectomía", en la cual puede dejarse parte del sigmoides, hasta que el paciente se recupera de la cirugía inicial. En estos casos, la resección de éste se realizará en el momento de la reconstitución del tránsito algunos meses después ${ }^{15}$. La morbilidad y mortalidad de la operación de Hartmann para la perforación libre del divertículo, aún es considerable. La mortalidad acumulada en un total de 1.051 pacientes reportada en 54 estudios combinados que se llevaron a cabo entre 1966 y 2003 fue cercana al $19 \%$ y se asoció a un $24 \%$ de incidencia de infección de la herida operatoria y a un $10 \%$ de complicaciones de la ostomía ${ }^{16}$. A pesar de los avances en el cuidado intensivo, imagenología y tratamiento médico, la mortalidad de esta condición se ha mantenido estable en el tiempo ${ }^{17}$. El tránsito intestinal puede ser reestablecido generalmente 3-6 meses después de la primera cirugía ${ }^{18}$, aunque se ha reportado que, en aproximadamente entre el 30\% y $70 \%$ de los pacientes nunca se cierra la colostomía ${ }^{15,19-21}$. Es más, se considera que la reconstitución tras una operación de Hartmann continúa siendo un procedimiento electivo difícil ${ }^{22}$, con morbilidad significativa ${ }^{23}$ y que aun no cuenta con estudios clínicos aleatorizados que demuestren diferencias estadísticamente significativas entre el procedimiento laparoscópico y el convencional ${ }^{24,25}$.

Considerando la morbi-mortalidad significativa asociada a la operación de Hartmann y sus secuelas, algunos autores han sugerido que en situaciones seleccionadas puede ser posible la resección del segmento perforado y la anastomosis primaria ${ }^{26,27}$, para lo cual puede ser beneficioso el lavado colónico intraoperatorio previo a realizar la anastomosis ${ }^{28,29}$. 
Esta conducta persiste controversial y la mayoría de los cirujanos no la recomendarían en el caso de peritonitis generalizada por diverticulitis perforada. En los casos seleccionados en que es posible realizar la anastomosis primaria, se puede proteger ésta con una ileostomía proximal en asa. Esta conducta pareciera ser preferible a un Hartmann cuando el grado de contaminación intraoperatoria y las condiciones subyacentes del paciente así lo permiten. En estos casos, el uso de una ostomía desfuncionalizante sumado a la anastomosis colorrectal puede resultar en un buen balance entre la morbilidad postoperatoria, calidad de vida y posibilidad de una ostomía permanente ${ }^{30}$.

En un estudio publicado en 2011 por Trentiet et $a l^{31}$, a un total de 87 pacientes se les realizó cirugía de urgencias por diverticulitis complicada con peritonitis purulenta o fecaloídea difusa (Hinchey grado III y IV). A 60 de ellos (69\%) se les realizó operación de Hartmann mientras que resección con anastomosis primaria fue hecha en 27 pacientes (31\%). En el análisis multivariado, la resección con anastomosis primaria se asoció significativamente a menores complicaciones postoperatorias. Tres pacientes con resección y anastomosis primaria $(11,1 \%)$ desarrollaron filtración de la anastomosis clínica y necesitaron reoperación. Por lo tanto, concluyen que la resección con anastomosis primaria puede realizarse de manera segura sin agregar morbilidad o mortalidad en casos de peritonitis difusa de origen diverticular. La operación de Hartmann debe reservarse solamente para pacientes hemodinámicamente inestables o de alto riesgo. La especialización en cirugía colorrectal disminuye la mortalidad y aumenta el porcentaje de cirugías en un tiempo.

El lavado peritoneal laparoscópico (LPL), constituye una nueva aproximación quirúrgica frente a la peritonitis generalizada. La llegada de la cirugía laparoscópica y el aumento del uso de ésta en el tratamiento de úlceras pépticas perforadas y apendicitis, ha llevado al desarrollo de estrategias laparoscópicas para el tratamiento de la diverticulitis perforada. En este contexto, el LPL es una alternativa terapéutica recientemente propuesta que podría potencialmente evitar al paciente una resección de colon y ostomía. Este procedimiento consiste en la irrigación profusa de la cavidad peritoneal asociada a una succión laparoscópica del líquido perfundido, la que es seguida por la inserción de un drenaje aspirativo en la región abdominal cercana al colon sigmoides ${ }^{29,32-36}$. Cualquier perforación que presente el colon se deja intacta, porque es cubierta de manera espontánea por el omento. La experiencia inicial ha sido prometedora con respecto a la mortalidad perioperatoria y las complicaciones ${ }^{30}$. Es más, si bien la mayoría de los que proponen el LPL inicial, proponen una sigmoidectomía electiva diferida ${ }^{33,37-40}$, un estudio multicéntrico irlandés reportó alentadores resultados luego del lavado seguido de una observación continua. De hecho, Myers ${ }^{41}$, encontró recurrencia de diverticulitis de sigmoides en 4 de 92 pacientes tratados, ninguno de los cuales requirieron cirugía en el seguimiento de hasta 36 meses. En un artículo de revisión de la literatura entre el año 1990 y 2008, Alamili et $a l^{42}$, concluyen que la mayoría de los pacientes con diverticulitis Hinchey III pueden ser manejados efectivamente mediante LPL en la fase aguda. La tasa de conversión a laparotomía fue de $3 \%$, promedio de estadía hospitalaria de 9 días, $10 \%$ de los pacientes presentaron complicaciones y hubo una mortalidad general de $1,4 \%$.

Estos datos de diferentes centros sugieren que el LPL tiene el potencial de convertirse, al menos en casos seleccionados, en el tratamiento definitivo para la diverticulitis perforada. Sin embargo, la información sobre el LPL para la peritonitis de origen diverticular aun es limitada y es necesaria mayor investigación para garantizar y confirmar estos resultados iniciales prometedores.

\section{Conclusiones}

El nuevo manejo a través de LPL asociado a tratamiento antibiótico endovenoso aparentemente tiene una baja tasa de morbilidad, baja mortalidad, corta estadía hospitalaria y puede ser realizada sin necesidad de colostomía. Otras ventajas comparadas con la operación de Hartmann son el menor tiempo quirúrgico y menores costos económicos. Así, el LPL sin resección de sigmoides en el cuadro agudo de peritonitis purulenta por una diverticulitis perforada puede ser considerado como una alternativa terapéutica válida a procedimientos más radicales que incluyen la operación de Hartmann. Sin embargo, es necesario investigar de manera minuciosa, especificar las indicaciones pre e intraoperatorias y si la resección electiva de colon se debe realizar a todos o algunos pacientes seleccionados en el seguimiento; para ello se necesitan ensayos clínicos válidos antes que se puedan establecer indicaciones precisas al respecto.

\section{Referencias}

1. Parks T. Natural history of diverticular disease of the colon. Clin Gastroenterol. 1975;4:53-69.

2. Parks T. Natural history of diverticular disease of the colon. A review of 521 cases. Br Med J. 1969;4:639-42

3. Young-Fadok T, Roberts P, Spencer M, Wolff B. Colonic diverticular disease. Curr Probl Surg. 2000;37:457516. 
4. Narula N, Marshall J. Role of probiotics in management of diverticular disease. J Gastroenterol Hepatol. 2010;25:1827-30.

5. Waugh J, Walt A. Current trends in the surgical treatment of diverticulitis of the sigmoid colon. Surg Clin North Am. 1962;42:1267-76.

6. Etzioni D, Mack T, Beart R Jr, Kaiser A. Diverticulitis in the United States: 1998-2005: changing patterns of disease and treatment. Ann Surg. 2009;249:210-7.

7. Mueller M, Glatzle J, Kasparek M, Becker H, Jehle $\mathrm{E}$, Zittel T, et al. Long-term outcome of conservative treatment in patients with diverticulitis of the sigmoid colon. Eur J Gastroenterol Hepatol. 2005; 17:649-54.

8. Sarin S, Boulos P. Long-term outcome of patients presenting with acute complications of diverticular disease. Ann R Coll Surg Engl. 1994;76:117-20.

9. Lohrmann C, Ghanem N, Pache G, Makowiec F, Kotter E, Langer M. CT in acute perforated sigmoid diverticulitis. European Journal of Radiology 2005;56:78-83.

10. Stollman N, Raskin J. Diverticular disease of the colon. Lancet 2004;363:631-9.

11. Nelson R, Ewing B, Wengert T, Thorson A. Clinical outcomes of complicated diverticulitis managed non operatively. Am J Surg. 2008;196:969-72.

12. Chouillard E, Maggiori L, Ata T, Jarbaoui S, Rivkine E, Benhaim L, et al. Laparoscopic two-stage left colonic resection for patients with peritonitis caused by acute diverticulitis. Dis Colon Rectum 2007;50:1157-63.

13. Agaba E, Zaidi R, Ramzy P, Aftab M, Rubach E, Gecelter $\mathrm{G}$, et al. Laparoscopic Hartmann's procedure: a viable option for treatment of acutely perforated diverticultis. Surg Endosc. 2009;23:1483-6.

14. Church J. Surgical treatment of sigmoid diverticulitis. Schweiz Med Wochenschr. 1991;121:744-8.

15. Salem L, Anaya D, Roberts K, Flum D. Hartmann's colectomy and reversal in diverticulitis: a populationlevel assessment. Dis Colon Rectum 2005;48:988-95.

16. Salem L, Flum D. Primary anastomosis or Hartmann's procedure for patients with diverticular peritonitis? A systematic review. Dis Colon Rectum 2004;47:1953-64.

17. Chandra V, Nelson H, Larson D, Harrington J. Impact of primary resection on the outcome of patients with perforated diverticulitis. Arch Surg. 2004;139:1221-4.

18. Oomen J, Cuesta M, Engel A. Reversal of Hartmann's procedure after surgery for complications of diverticular disease of the sigmoid colon is safe and possible in most patients. Dig Surg. 2005;22:419-25.

19. Elliott T, Yego S, Irvin T. Five-year audit of the acute complications of diverticular disease. Br J Surg. 1997;84:535-9.

20. Wedell J, Banzhaf G, Chaoui R, Fischer R, Reichmann J. Surgical management of complicated colonic diverticulitis. Br J Surg. 1997;84:380-3.

21. Maggard M, Zingmond D, O'Connell J, Ko C. What proportion of patients with an ostomy (for diverticulitis) get reversed? Am Surg. 2004;70:928-31.
22. Wigmore S, Duthie G, Young I, Spalding E, Rainey J. Restoration of intestinal continuity following Hartmann's procedure: the Lothian experience 19871992. Br J Surg. 1995;82:27-30.

23. Aydin H, Remzi F, Tekkis P, Fazio V. Hartmann's reversal is associated with high postoperative adverse events. Dis Colon Rectum 2005;48:2117-26.

24. Caselli C, Bambs C, Pinedo G, Molina M, Zuñiga A, Bellolio F. Laparoscopic approach for intestinal passage reconstruction after Hartmann's operation: experience with 30 patients. Cir Esp. 2010;88:314-8.

25. Lee E, Murray J, Coller J, Roberts P, Schoetz D Jr. Intraoperative colonic lavage in nonelective surgery for diverticular disease. Dis Colon Rectum 1997;40:669-74.

26. Richter S, Lindemann W, Kollmar O, Pistorius G, Maurer C, Schilling M. One-stage sigmoid colon resection for perforated sigmoid diverticulitis (Hinchey stages III and IV). World J Surg. 2006;30:1027-32.

27. Abbas S. Resection and primary anastomosis in acute complicated diverticulitis, a systematic review of the literature. Int J Colorectal Dis. 2007;22:351-7.

28. Biondo S, Perea M, Rague J, Parés D, Jaurrieta E. Onestage procedure in non-elective surgery for diverticular disease complications. Colorectal Dis. 2001;3:42-5.

29. Regenet N, Tuech J, Pessaux P, Ziani M, Rouge C, Hennekinne $\mathrm{S}$, et al. Intraoperative colonic lavage with primary anastomosis vs. Hartmann's procedure for perforated diverticular disease of the colon: a consecutive study. Hepatogastroenterol. 2002;49:664-7.

30. Constantinides V, Heriot A, Remzi F, Darzi A, Senapati A, Fazio V, et al. Operative strategies for diverticular peritonitis: a decision analysis between primary resection and anastomosis versus Hartmann's procedures. Ann Surg. 2007;245:94-103.

31. Trenti L, Biondo S, Golda T, Monica M, Kreisler E, Fraccalvieri D, et al. Generalized peritonitis due to perforated diverticulitis: Hartmann's procedure or primary anastomosis? Int J Colorectal Dis. 2011;26:377-84.

32. O'Sullivan G, Murphy D, O'Brien M, Ireland A. Laparoscopic management of generalized peritonitis due to perforated colonic diverticula. Am J Surg. 1996;171:432-4.

33. Faranda C, Barrat C, Catheline J, Champault G. Two stage laparoscopic management of generalized peritonitis due to perforated sigmoid diverticula: eighteen cases. Surg Laparosc Endosc Percutan Tech. 2000;10:135-8.

34. Greco R, Kamath C, Nosher J. Percutaneous drainage of peridiverticular abscess followed by primary sigmoidectomy. Dis Colon Rectum 1982;25:53-5.

35. Saini S, Mueller P, Wittenberg J, Butch R, Rodkey G, Welch C. Percutaneous drainage of diverticular abscesses. Arch Surg. 1986;121:475-8.

36. Da Rold A, Guerriero S, Fiamingo P, Pariset S, Veroux $\mathrm{M}$, Pilon $\mathrm{F}$, et al. Laparoscopic colorrhaphy, irrigation and drainage in the treatment of complicated acute diverticulitis: initial experience. Chir Ital. 2004;56:95-8. 
37. Taylor C, Layani L, Ghusn M, White S. Perforated diverticulitis managed by laparoscopic lavage. ANZ J Surg. 2006;76:962-5.

38. Bretagnol F, Pautrat K, Mor C, Benchellal Z, Huten $\mathrm{N}$, de Calan L. Emergency laparoscopic management of perforated sigmoid diverticulitis: a promising alternative to more radical procedures. J Am Coll Surg. 2008;206:654-7.

39. Franklin M Jr, Portillo G, Trevino J, Gonzalez J, Glass J. Long-term experience with the laparoscopic approach to perforated diverticulitis plus generalized peritonitis. World J Surg. 2008;32:1507-11.
40. Karoui M, Champault A, Pautrat K, Valleur P, Cherqui D, Champault G. Laparoscopic peritoneal lavage or primary anastomosis with defunctioning stoma for Hinchey 3 complicated diverticulitis: results of a comparative study. Dis Colon Rectum 2009;52:609-15.

41. Myers E, Hurley M, O’Sullivan G, Kavanagh D, Wilson I, Winter D. Laparoscopic peritoneal lavage for generalized peritonitis due to perforated diverticulitis. $\mathrm{Br} \mathrm{J}$ Surg. 2008;95:97-101.

42. Alamili M, Gögenur I, Rosenberg J. Acute complicated diverticulitis managed by laparoscopic lavage. Dis Colon Rectum 2009;52:1345-9. 